\title{
Physical properties and transmission spectrum of the WASP-80 planetary system from multi-colour photometry ${ }^{\star}$
}

L. Mancini ${ }^{1}$, J. Southworth ${ }^{2}$, S. Ciceri ${ }^{1}$, M. Dominik ${ }^{3}$, Th. Henning ${ }^{1}$, U. G. Jørgensen ${ }^{4,5}$, A. F. Lanza ${ }^{6}$, M. Rabus ${ }^{7,1}$, C. Snodgrass ${ }^{8}$, C. Vilela ${ }^{2}$, K. A. Alsubai ${ }^{9}$, V. Bozza ${ }^{10,11}$, D. M. Bramich ${ }^{12}$, S. Calchi Novati ${ }^{13,10}$, G. D’ Ago $^{10,11}$, R. Figuera Jaimes ${ }^{14,3}$, P. Galianni ${ }^{3}$, S.-H. Gu ${ }^{15,16}$, K. Harpsøe $\mathrm{e}^{4,5}$, T. Hinse ${ }^{17}$, M. Hundertmark ${ }^{3}$, D. Juncher ${ }^{4,5}$, N. Kains ${ }^{14}$, H. Korhonen ${ }^{18,4,5}$, A. Popovas ${ }^{4,5}$, S. Rahvar ${ }^{19,20}$, J. Skottfelt ${ }^{4,5}$, R. Street ${ }^{21}$, J. Surdej ${ }^{22}$, Y. Tsapras ${ }^{21,23}$, X.-B. Wang ${ }^{15,16}$, and O. Wertz ${ }^{22}$

1 Max Planck Institute for Astronomy, Königstuhl 17, 69117 Heidelberg, Germany e-mail: mancini@mpia.de

2 Astrophysics Group, Keele University, Keele, ST5 5BG, UK

3 SUPA, University of St Andrews, School of Physics \& Astronomy, North Haugh, St Andrews KY16 9SS, UK

${ }^{4}$ Niels Bohr Institute, University of Copenhagen, Juliane Maries vej 30, 2100 Copenhagen $\varnothing$, Denmark

5 Centre for Star and Planet Formation, Geological Museum, Øster Voldgade 5-7, 1350 Copenhagen, Denmark

${ }^{6}$ INAF-Osservatorio Astrofisico di Catania, via S.Sofia 78, 95123 Catania, Italy

7 Instituto de Astrofísica, Facultad de Física, Pontificia Universidad Católica de Chile, Av. Vicuña Mackenna 4860, 7820436 Macul, Santiago, Chile

8 Max-Planck-Institute for Solar System Research, Max-Planck Str. 2, 37191 Katlenburg-Lindau, Germany

9 Qatar Foundation, PO Box 5825, Doha, Qatar

10 Dipartimento di Fisica "E.R. Caianiello", University of Salerno, via Giovanni Paolo II, 84084 Fisciano, Italy

11 Istituto Nazionale di Fisica Nucleare, Sezione di Napoli, Napoli, Italy

12 Qatar Environment and Energy Research Institute, Qatar Foundation, Tornado Tower, Floor 19, PO Box 5825, Doha, Qatar

13 Istituto Internazionale per gli Alti Studi Scientifici (IIASS), 84019 Vietri Sul Mare, Italy

14 European Southern Observatory, Karl-Schwarzschild-Straße 2, 85748 Garching bei München, Germany

15 Yunnan Observatory, Chinese Academy of Sciences, 650011 Kunming, PR China

16 Key Laboratory for the Structure and Evolution of Celestial Objects, Chinese Academy of Sciences, 650011 Kunming, PR China

17 Korea Astronomy and Space Science Institute, 305-348 Daejeon, Republic of Korea

18 Finnish Centre for Astronomy with ESO (FINCA), University of Turku, Väisäläntie 20, 21500 Piikkiö, Finland

19 Department of Physics, Sharif University of Technology, PO Box 11155-9161 Tehran, Iran

20 Perimeter Institute for Theoretical Physics, 31 Caroline St. N., Waterloo N2L 2Y5,Canada

21 Las Cumbres Observatory Global Telescope Network, 6740B Cortona Drive, Goleta CA 93117, USA

${ }^{22}$ Institut d'Astrophysique et de Géophysique, Université de Liège, 4000 Liège, Belgium

23 School of Physics and Astronomy, Queen Mary University of London, Mile End Road, London E1 4NS, UK

Received 17 December 2013 / Accepted 18 January 2014

\section{ABSTRACT}

\begin{abstract}
WASP-80 is one of only two systems known to contain a hot Jupiter which transits its M-dwarf host star. We present eight light curves of one transit event, obtained simultaneously using two defocussed telescopes. These data were taken through the Bessell $I$, Sloan $g^{\prime} r^{\prime} i^{\prime} z^{\prime}$ and near-infrared $J H K$ passbands. We use our data to search for opacity-induced changes in the planetary radius, but find that all values agree with each other. Our data are therefore consistent with a flat transmission spectrum to within the observational uncertainties. We also measure an activity index of the host star of $\log R_{\mathrm{HK}}^{\prime}=-4.495$, meaning that WASP-80 A shows strong chromospheric activity. The non-detection of starspots implies that, if they exist, they must be small and symmetrically distributed on the stellar surface. We model all available optical transit light curves and obtain improved physical properties and orbital ephemerides for the system.
\end{abstract}

Key words. planetary systems - stars: fundamental parameters - stars: individual: WASP- 80 - techniques: photometric

\section{Introduction}

Planetary systems in which the host star is a late-type dwarf are of particular interest because they have favourable ratios of planetary mass and radius to those of the star. This makes the detections of small and low-mass planets easier for the transit

* Full Table 2 is only available at the CDS via anonymous ftp to cdsarc.u-strasbg.fr (130.79.128.5) or via http://cdsarc.u-strasbg.fr/viz-bin/qcat?]/A+A/562/A126 and Doppler methods, respectively. If the planet is transiting and the parent star is bright, then the planetary atmosphere can be probed by transmission spectroscopy (e.g. GJ 1214 b: Croll et al. 2011; Crossfield et al. 2011; Bean et al. 2011; Berta et al. 2012; Colòn \& Gaidos 2013; GJ 3470 b: Crossfield et al. 2013; GJ 436 b: Pont et al. 2009; Gibson et al. 2011), transmission photometry (e.g. GJ 1214 b: de Mooij et al. 2012; Murgas et al. 2012; de Mooij et al. 2013; Narita et al. 2013; GJ 3470 b: Nascimbeni et al. 2013) and observations of secondary eclipses 
Table 1. Details of the transit observations presented in this work.

\begin{tabular}{lccccccccccc}
\hline \hline Telescope & $\begin{array}{c}\text { Date of } \\
\text { first obs. }\end{array}$ & $\begin{array}{c}\text { Start time } \\
(\mathrm{UT})\end{array}$ & $\begin{array}{c}\text { End time } \\
(\mathrm{UT})\end{array}$ & $N_{\mathrm{obs}}$ & $\begin{array}{c}T_{\text {exp }} \\
(\mathrm{s})\end{array}$ & $\begin{array}{c}T_{\text {obs }} \\
(\mathrm{s})\end{array}$ & Filter & Airmass & $\begin{array}{c}\text { Moon } \\
\text { illum. }\end{array}$ & $\begin{array}{c}\text { Aperture } \\
\text { radii }(\mathrm{px})\end{array}$ & $\begin{array}{c}\text { Scatter } \\
(\mathrm{mmag})\end{array}$ \\
\hline DFOSC & 20130616 & $05: 35$ & $09: 44$ & 200 & 60 & 75 & Bessel $I$ & $1.33 \rightarrow 1.12 \rightarrow 1.37$ & $46 \%$ & $16,38,60$ & 0.49 \\
GROND & 20130616 & $05: 00$ & $10: 50$ & 162 & 60 & 120 & Sloan $g^{\prime}$ & $1.38 \rightarrow 1.12 \rightarrow 1.79$ & $46 \%$ & $30,90,120$ & 0.80 \\
GROND & 20130616 & $05: 00$ & $10: 50$ & 162 & 60 & 120 & Sloan $r^{\prime}$ & $1.38 \rightarrow 1.12 \rightarrow 1.79$ & $46 \%$ & $35,100,120$ & 0.49 \\
GROND & 20130616 & $05: 00$ & $10: 50$ & 162 & 60 & 120 & Sloan $i^{\prime}$ & $1.38 \rightarrow 1.12 \rightarrow 1.79$ & $46 \%$ & $35,80,100$ & 0.82 \\
GROND & 20130616 & $05: 00$ & $10: 50$ & 162 & 60 & 120 & Sloan $z^{\prime}$ & $1.38 \rightarrow 1.12 \rightarrow 1.79$ & $46 \%$ & $30,80,100$ & 1.06 \\
GROND & 20130616 & $05: 00$ & $10: 50$ & 523 & 4 & 38 & $J$ & $1.38 \rightarrow 1.12 \rightarrow 1.79$ & $46 \%$ & $6,11,21$ & 4.15 \\
GROND & 20130616 & $05: 00$ & $10: 50$ & 523 & 4 & 38 & $H$ & $1.38 \rightarrow 1.12 \rightarrow 1.79$ & $46 \%$ & $5,12,22$ & 3.28 \\
GROND & 20130616 & $05: 00$ & $10: 50$ & 523 & 4 & 38 & $K$ & $1.38 \rightarrow 1.12 \rightarrow 1.79$ & $46 \%$ & $7,11,20$ & 5.14 \\
\hline
\end{tabular}

Notes. $N_{\mathrm{obs}}$ is the number of observations, $T_{\text {exp }}$ is the exposure time, $T_{\mathrm{obs}}$ is the observational cadence, and "Moon illum." is the fractional illumination of the Moon at the midpoint of the transit. The aperture sizes are the radii of the software apertures for the star, inner sky and outer sky, respectively. Scatter is the rms scatter of the data versus a fitted model.

(e.g. GJ 1214 b: Fraine et al. 2013; GJ 436 b: Stevenson et al. 2010; Knutson et al. 2011).

Recent analyses of HARPS and Kepler data suggest that Neptunes and super-Earths with orbital periods shorter than 50 days are very abundant around $\mathrm{M}$ stars (Bonfils et al. 2013; Dressing \& Charbonneau 2013). Bonfils et al. (2013) also established that giant planets have a much lower occurrence rate for orbital periods in the range 10-100d, supporting the idea that the frequency of giant planets decreases toward less massive parent stars, irrespective of period (Johnson et al. 2010).

Accordingly, only two transiting hot Jupiters have so far been found orbiting $\mathrm{M}$ dwarfs ${ }^{1}$. These are Kepler-45 b (KOI$254 ; R_{\mathrm{p}}=0.999 R_{\mathrm{Jup}}, M_{\mathrm{p}}=0.500 M_{\mathrm{Jup}}$, Johnson et al. 2012; Southworth 2012) and WASP-80b $\left(R_{\mathrm{p}}=0.95 R_{\mathrm{Jup}}, M_{\mathrm{p}}=\right.$ $0.55 M_{\text {Jup }}$, Triaud et al. 2013). Whilst Kepler-45 is a distant (333 pc) and faint $(V=16.9)$ star, WASP-80 is much closer $(60 \mathrm{pc})$ and brighter $(V=11.9)$, enabling a detailed study of its characteristics with ground-based facilities. Moreover, due to its low density and large transit depth $(\sim 3 \%)$, WASP-80 b is a very suitable target for transmission spectroscopy and photometry.

Here we present photometric observations of a transit of WASP-80 b, observed simultaneously with two telescopes and in eight different passbands. We use these data to refine the physical parameters of the planetary system and provide the first probe of the day-night terminator region of this giant planet by transmission photometry.

\section{Observations and data reduction}

A complete transit of WASP-80 b was observed on 2013 June 16 (see Table 1), using the DFOSC imager mounted on the $1.54-\mathrm{m}$ Danish Telescope at ESO La Silla during the 2013 observing campaign by the MiNDSTEp consortium (Dominik et al. 2010). The instrument has a field of view of $13.7^{\prime} \times 13.7^{\prime}$ and a plate scale of $0.39^{\prime \prime}$ pixel $^{-1}$. The observations were performed through a Bessel $I$ filter and using the defocussing method. The telescope was autoguided and the CCD was windowed to reduce the readout time. The night was photometric.

The data were reduced using DEFOT, an $\mathrm{IDL}^{2}$ pipeline for time-series photometry (Southworth et al. 2009). The images were debiased and flat-fielded using standard methods, then

\footnotetext{
1 Two brown dwarfs are also known to transit M stars: NLTT 41135 (Irwin et al. 2010) and LHS 6343 (Johnson et al. 2011).

2 IDL is a trademark of the ITT Visual Information Solutions: http://www.ittvis. com/ProductServices/IDL . aspx
}

subjected to aperture photometry using the $\mathrm{APER}^{3}$ task and an optimal ensemble of comparison stars. Pointing variations were followed by cross-correlating each image against a reference image. The shape of the light curve is very insensitive to the aperture sizes, so we chose those which yielded the lowest scatter. The final light curve was detrended to remove slow instrumental and astrophysical trends by fitting a straight line to the out-oftransit data. This process was simultaneous with the optimisation of the weights of an ensemble of comparison stars. The final differential-flux light curve is plotted in Fig. 1.

The same transit was also observed using the Gamma Ray burst Optical and Near-infrared Detector (GROND) instrument mounted on the $\mathrm{MPG}^{4} / \mathrm{ESO} 2.2-\mathrm{m}$ telescope, also located at ESO La Silla. GROND is an imaging system capable of simultaneous photometric observations in four optical (similar to Sloan $\left.g^{\prime}, r^{\prime}, i^{\prime}, z^{\prime}\right)$ and three near-infrared (NIR; $J, H, K$ ) passbands (Greiner et al. 2008). Each of the four optical channels is equipped with a back-illuminated $2048 \times 2048$ E2V CCD, with a field of view of $5.4^{\prime} \times 5.4^{\prime}$ at $0.158^{\prime \prime}$ pixel $^{-1}$. The three NIR channels use $1024 \times 1024$ Rockwell HAWAII-1 arrays with a field of view of $10^{\prime} \times 10^{\prime}$ at $0.6^{\prime \prime}$ pixel $^{-1}$. The observations were also performed with the telescope defocussed.

The optical data were reduced as for the Danish Telescope, except that a quadratic function was used to detrend the light curves. The NIR data were reduced following the procedure described in Mancini et al. (2013c). The optical light curves are plotted superimposed in the bottom panel of Fig. 1 in order to highlight the differences among the transit depths and light curve shapes of the simultaneous multi-band observations. The GROND $i^{\prime}$ and Danish Telescope $I$ light curves are in excellent agreement. The differential-magnitude light curves are given in Table 2.

\subsection{Stellar activity measurement}

We obtained a spectrum of the $\mathrm{Ca}$ II $\mathrm{H}$ and $\mathrm{K}$ lines on the night of 2012 October 1, using the William Herschel Telescope with the ISIS grating spectrograph, in order to measure the $\log R_{\mathrm{HK}}^{\prime}$ stellar activity index. With the H2400B grating we obtained a spectrum covering $375-415 \mathrm{~nm}$ at a reciprocal dispersion of $0.011 \mathrm{~nm} \mathrm{pixel}^{-1}$. An exposure time of $600 \mathrm{~s}$ yielded a continuum signal to noise ratio of approximately 20 in the region of the $\mathrm{H}$ and $\mathrm{K}$ lines. The data were reduced using optimal

\footnotetext{
3 APER is part of the ASTROLIB subroutine library distributed by NASA on http: //idlastro.gsfc.nasa.gov

4 Max Planck Gesellschaft.
} 

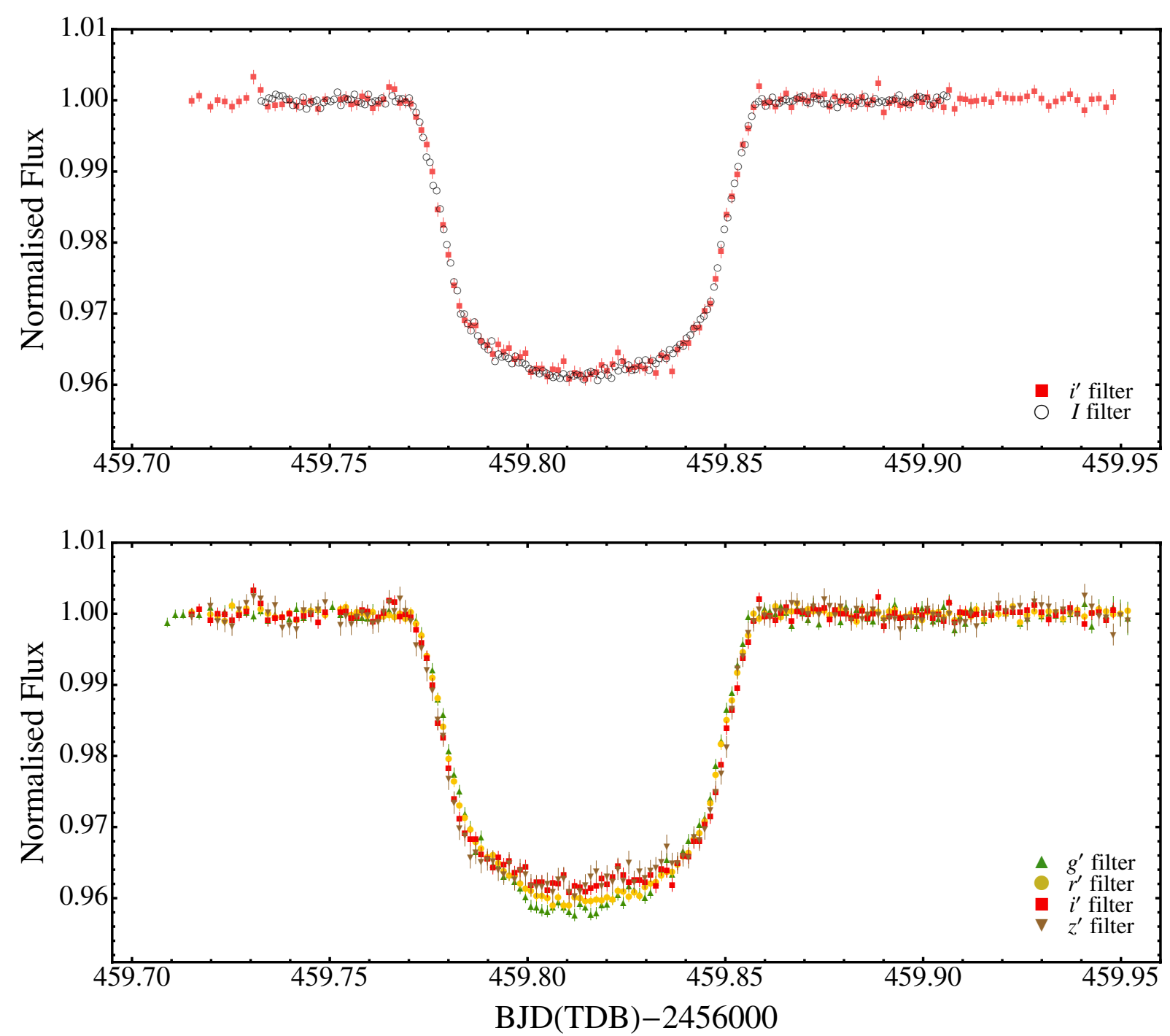

Fig. 1. Light curves of a transit of WASP-80 b. Top panel: light curves obtained with the Danish Telescope (Bessel-I filter) and with GROND $\left(\right.$ Sloan $\left.-i^{\prime}\right)$, highlighting the good match between the transit shapes in the two independent observations. The circles denoting the DK points have the same size as the corresponding error bars, which have been suppressed for clarity. Bottom panel: light curves obtained with GROND through four optical filters simultaneously, showing how the transit shape changes with wavelength.

Table 2. Excerpts of the light curves of WASP-80.

\begin{tabular}{lccrc}
\hline \hline Telescope & Filter & BJD (TDB) & Diff. mag. & Uncertainty \\
\hline DK 1.54-m & $I$ & 2456459.732729 & 0.00002 & 0.00055 \\
DK 1.54-m & $I$ & 2456459.733794 & 0.00029 & 0.00055 \\
ESO 2.2-m & $g^{\prime}$ & 2456459.708924 & 0.00122 & 0.00087 \\
ESO 2.2-m & $g^{\prime}$ & 2456459.710953 & 0.00079 & 0.00087 \\
ESO 2.2-m & $r^{\prime}$ & 2456459.715126 & -0.00021 & 0.00033 \\
ESO 2.2-m & $r^{\prime}$ & 2456459.719854 & 0.00084 & 0.00033 \\
ESO 2.2-m & $i^{\prime}$ & 2456459.715126 & 0.00009 & 0.00064 \\
ESO 2.2-m & $i^{\prime}$ & 2456459.716988 & -0.00059 & 0.00064 \\
ESO 2.2-m & $z^{\prime}$ & 2456459.715126 & 0.00041 & 0.00064 \\
ESO 2.2-m & $z^{\prime}$ & 2456459.719854 & -0.00109 & 0.00064 \\
\hline
\end{tabular}

Notes. Full Table 2 is available at the CDS. A portion is shown here for guidance regarding its form and content.

extraction as implemented in the PAMELA and MOLLY packages (Marsh 1989) and calibrated onto the Mt. Wilson system using 20 standard stars from Vaughan et al. (1978); further details can be found in Vilela et al. (in prep.).
The very strong $\mathrm{H}$ and $\mathrm{K}$ emission lines for WASP-80 A (Fig. 2) yield the emission measure $\log R_{\mathrm{HK}}^{\prime}=-4.495$, which is indicative of high activity (e.g. Noyes et al. 1984). Given this strong chromospheric emission one might expect to see evidence of spot activity, but Triaud et al. (2013) found no rotational modulation in the SuperWASP light curves to a limit of $\sim 1 \mathrm{mmag}$, and we see no evidence of spot anomalies (e.g. Tregloan-Reed et al. 2013) in our light curves. A plausible explanation is that the stellar surface contains many spots which are too small to noticeably affect transit light curves, and which are approximately symmetrically distributed so cause no measurable rotation signal in the SuperWASP light curves.

This suggestion is in agreement with the conclusions of Jackson \& Jeffries (2012) (see also Jackson \& Jeffries 2013), who analysed two large samples of low-mass stars $(0.2<$ $\left.M_{\star} / M_{\odot}<0.7\right)$ in the open cluster NGC 2516: one sample with measurable rotational modulation and one without. The two samples coincide on the colour-magnitude diagram for the cluster, and have the same rotational velocities and levels of chromospheric activity. This difference can be explained by the photometrically constant stars having many small starspots rather than few large ones. 


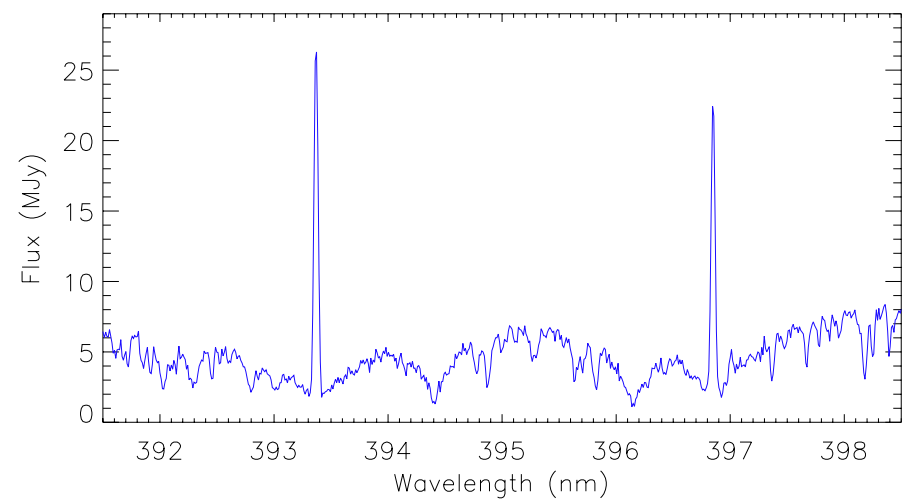

Fig. 2. Spectrum of WASP-80 in the region of the Ca II H and $\mathrm{K}$ lines showing the strong chromospheric emission in the line cores.

In the case of WASP-80, assuming that the photometric modulation due to starspots is smaller than $1 \mathrm{mmag}$, we can estimate the maximum deviation of the covering factor from uniformity of a few $10^{-3}$ of the disc area, by considering that the spot temperature contrast is probably smaller in cooler stars with respect to the Sun (see Berdyugina 2005).

\section{Light-curve analysis}

The light curves were modelled using the JKTEBOP ${ }^{5}$ code (see Southworth 2012, and references therein), which represents the star and planet as biaxial spheroids for calculation of the reflection and ellipsoidal effects and as spheres for calculation of the eclipse shapes. The main parameters fitted by JKTEBOP are the orbital inclination, $i$, the transit midpoint, $T_{0}$, and the sum and ratio of the fractional radii of the star and planet, $r_{\mathrm{A}}+r_{\mathrm{b}}$ and $k=r_{\mathrm{b}} / r_{\mathrm{A}}$. The fractional radii are defined as $r_{\mathrm{A}}=R_{\mathrm{A}} / a$ and $r_{\mathrm{b}}=R_{\mathrm{b}} / a$, where $a$ is the orbital semimajor axis, and $R_{\mathrm{A}}$ and $R_{\mathrm{b}}$ are the absolute radii of the star and the planet, respectively. Each light curve was modelled separately using the quadratic limb darkening (LD) law. The linear LD coefficients were fitted to the data whereas the quadratic LD coefficients were fixed at theoretical values (Claret 2004b) but perturbed by \pm 0.1 during the process of error estimation. We assumed that the orbit was circular (Triaud et al. 2013). We included in the fits the coefficients of a linear (DFOSC) or quadratic (GROND) polynomial versus time in order to fully account for the uncertainty in the detrending of the light curves.

Time-series photometry is unavoidably afflicted by correlated (red) noise which is not accounted for by standard error estimation algorithms (e.g. Carter \& Winn 2009). The APER algorithm we use to perform aperture photometry also tends to underestimate the true uncertainties in the relative magnitude measurements. We therefore rescaled the error bars as in our previous works (Mancini et al. 2013a,b,c), first to give a reduced $\chi^{2}$ of $\chi_{v}^{2}=1$ and then using the $\beta$ approach (e.g. Gillon et al. 2006; Winn et al. 2008; Gibson et al. 2008).

\subsection{Orbital period determination}

We used our photometric data to refine the orbital period of WASP- $80 \mathrm{~b}$. The transit time for each of our datasets was obtained by fitting with JKTEBOP, and uncertainties were estimated

\footnotetext{
5 The source code of JKTEBOP is available at: http://www . astro. keele.ac.uk/jkt/codes/jktebop.html
}

Table 3. Times of mid-transit of WASP- $80 \mathrm{~b}$ and their residuals versus a linear orbital ephemeris.

\begin{tabular}{lrrc}
\hline \hline Time of minimum & $\begin{array}{r}\text { Cycle } \\
\text { No. }\end{array}$ & $\begin{array}{r}\text { Residual } \\
(\mathrm{d})\end{array}$ & Reference \\
\hline 5 JD(TDB) -2400000 & -23 & 0.000220 & 1 \\
\hline $56054.856812 \pm 0.000135$ & 3 & -0.000078 & 1 \\
$56180.620911 \pm 0.000222$ & 18 & -0.000233 & 1 \\
$56459.814384 \pm 0.000044$ & 109 & 0.000081 & 2 \\
$56459.814284 \pm 0.000096$ & 109 & -0.000019 & 3 \\
$56459.814414 \pm 0.000082$ & 109 & 0.000111 & 4 \\
$56459.814350 \pm 0.000103$ & 109 & 0.000047 & 5 \\
$56459.814233 \pm 0.000161$ & 109 & -0.000070 & 6 \\
\hline
\end{tabular}

Notes. References: (1) Triaud et al. (2013); (2) Danish telescope (this work); (3) GROND $g^{\prime}$ (this work); (4) GROND $r^{\prime}$ (this work); (5) GROND $i^{\prime}$ (this work); (6) GROND $z^{\prime}$ (this work).

using Monte Carlo simulations. We also modelled the followup light curves reported in Triaud et al. (2013) in order to obtain a timing for each dataset. All timings were placed on the BJD(TDB) time system and are summarised in Table 3. The resulting measurements of transit midpoints were fitted with a straight line to obtain a final orbital ephemeris:

$$
T_{0}=\mathrm{BJD}(\mathrm{TDB}) 2456125.417405(99)+3.06786144(87) E,
$$

where $E$ is the number of orbital cycles after the reference epoch, which we take to be that estimated by Triaud et al. (2013), and quantities in brackets denote the uncertainty in the final digit of the preceding number. The quality of fit, $\chi_{v}^{2}=0.99$, indicates that a linear ephemeris is a good match to the observations. A plot of the residuals (Fig. 3) shows no evidence for systematic deviations from the predicted transit times. However, the number of observed transits of this planet is still very low so transit timing variations cannot be ruled out.

\subsection{Photometric parameters}

The GROND light curves and the JKTEBOP best-fitting models are shown in Fig. 4. A similar plot is reported in Fig. 5 for the light curves from the Danish Telescope and from Triaud et al. (2013). The parameters of the fits are given in Table 4. Uncertainties in the fitted parameters from each solution were calculated from 3500 Monte Carlo simulations and by a residualpermutation algorithm (Southworth 2008). The larger of the two possible error bars was retained in each case. The final photometric parameters are the weighted mean of the results presented in Table 4. Values obtained by Triaud et al. (2013) are also reported for comparison. Due to their lower quality (see Fig.4), we did not use the GROND-NIR light curves to estimate the final photometric parameters of WASP-80.

\section{Physical properties}

Following the Homogeneous Studies approach (Southworth 2012, and references therein), we used the photometric parameters estimated in the previous section and the spectroscopic properties of the parent star (velocity amplitude $K_{\mathrm{A}}=$ $110.9_{-3.3}^{+3.0} \mathrm{~m} \mathrm{~s}^{-1}$, effective temperature $T_{\text {eff }}=4145 \pm 100 \mathrm{~K}$ and metallicity $\left[\frac{\mathrm{Fe}}{\mathrm{H}}\right]=-0.14 \pm 0.16$ ) taken from Triaud et al. (2013), to revise the physical properties of the WASP-80 system using the ABSDIM code.

We iteratively determined the velocity amplitude of the planet $\left(K_{\mathrm{b}}\right)$ which yielded the best agreement between the 


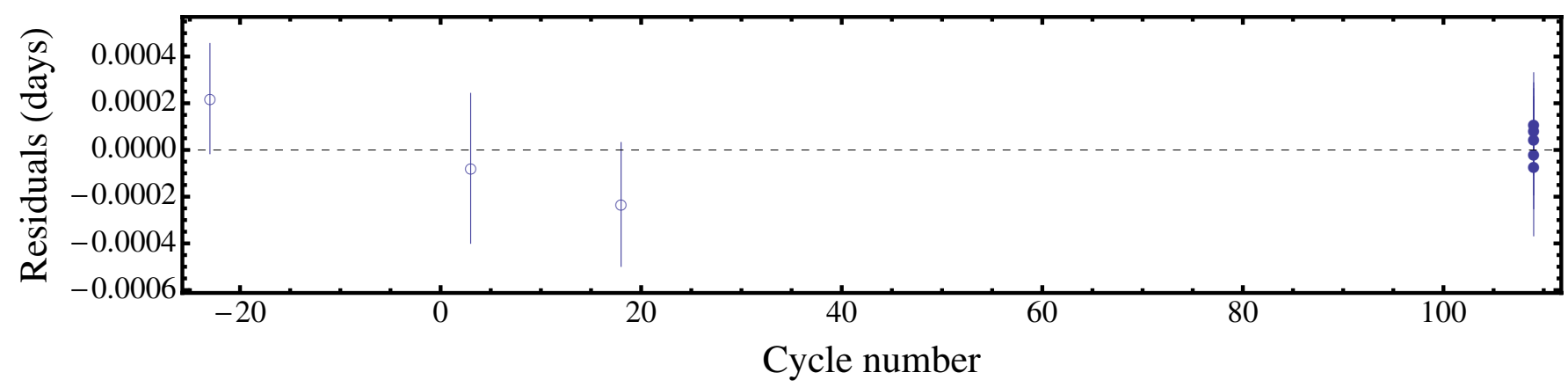

Fig. 3. Residuals of the timings of mid-transit of WASP- $80 \mathrm{~b}$ versus a linear ephemeris. Timings based on the observations obtained by Triaud et al. (2013) are plotted using open circles, while the other timings (this work) are plotted with filled circles.
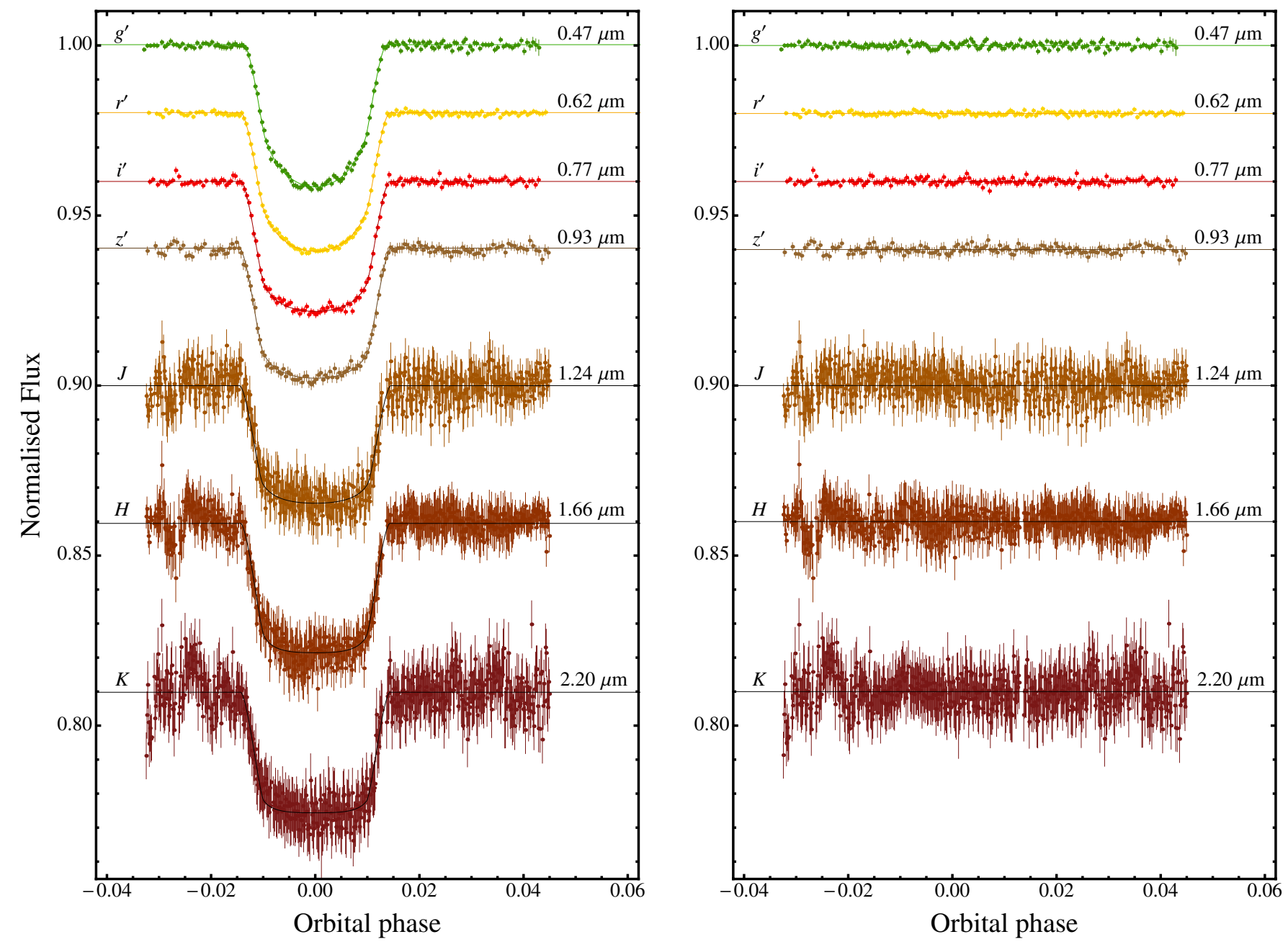

Fig. 4. Left-hand panel: simultaneous optical and NIR light curves of the transit event of WASP-80 b observed with GROND. The JKTEBOP best fits are shown as solid lines for each data set. The passbands are labelled on the left of the figure, and their central wavelengths are given on the right. Right-hand panel: residuals of each fit.

measured $R_{\mathrm{A}} / a$ and $T_{\text {eff }}$, and those predicted by a set of theoretical stellar models for the calculated stellar mass and $\left[\frac{\mathrm{Fe}}{\mathrm{H}}\right]$. The overall best fit was found over a grid of ages extending from the zero-age main sequence to a maximum of $5 \mathrm{Gyr}$, imposed because WASP-80 A shows strong activity indicative of youth (e.g. West et al. 2008). Statistical errors were propagated by a perturbation analysis. Systematic errors were estimated by calculating sets of results using five different sets of theoretical models (Claret 2004a; Demarque et al. 2004; Pietrinferni et al. 2004; VandenBerg et al. 2006; Dotter et al. 2008). The five models were given equal relative weighting. The resulting estimates of the physical properties are given in Table 5. For completeness we also estimated the physical properties of the WASP- 80 system using empirical calibrations based on detached eclipsing binary systems, instead of theoretical stellar models, using the method proposed by Enoch et al. (2010) and the calibration equations by Southworth (2010). Table 5 shows that the system properties obtained using the empirical calibration and the theoretical model sets agree well, except for the models by Claret (2004a). This discrepancy can be attributed to the differences in the $T_{\text {eff }}$ scale 

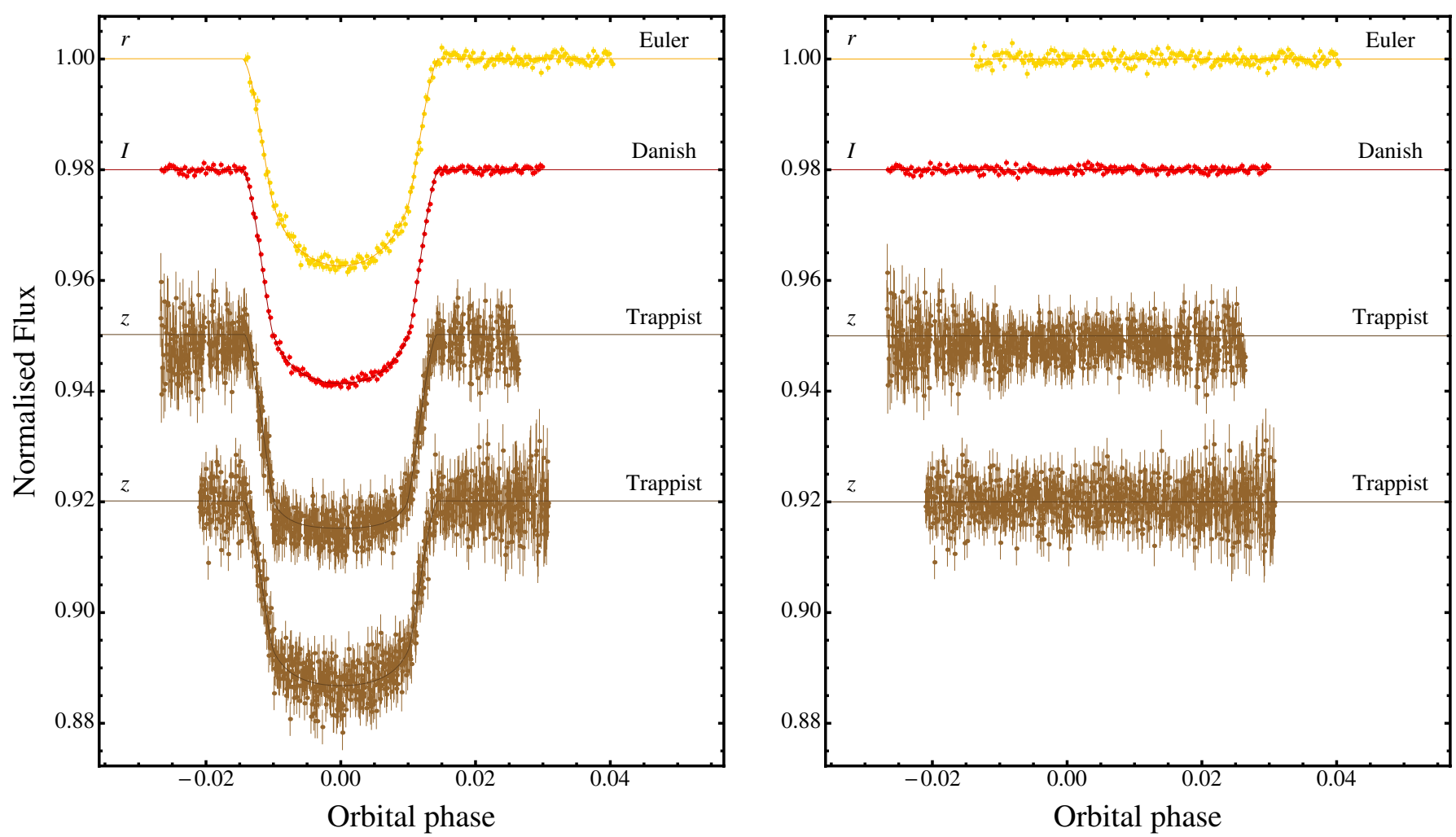

Fig. 5. Left-hand panel: light curves of transit events of WASP-80 b observed in Gunn $r$ with the Euler telescope (Triaud et al. 2013), in Bessell $I$ with the Danish telescope (this work) and in $z$ with TRAPPIST (Triaud et al. 2013). The light curves are ordered according to central wavelength of the filter used. The JKTEBOP best fits are shown as solid lines for each optical data set. Right-hand panel: residuals of each fit.

Table 4. Parameters of the JKTEBOP fits to the light curves of WASP-80.

\begin{tabular}{llccccc}
\hline \hline Telescope & Filter & $r_{\mathrm{A}}+r_{\mathrm{b}}$ & $k$ & $i^{\circ}$ & $r_{\mathrm{A}}$ & $r_{\mathrm{b}}$ \\
\hline Danish 1.54-m & Bessel $I$ & $0.09324 \pm 0.00095$ & $0.17135 \pm 0.00099$ & $88.99 \pm 0.23$ & $0.07960 \pm 0.00074$ & $0.01364 \pm 0.00018$ \\
MPG/ESO 2.2-m & Sloan $g^{\prime}$ & $0.09131 \pm 0.00161$ & $0.17033 \pm 0.00217$ & $89.20 \pm 0.59$ & $0.07802 \pm 0.00128$ & $0.01329 \pm 0.00035$ \\
MPG/ESO 2.2-m & Sloan $r^{\prime}$ & $0.09001 \pm 0.00290$ & $0.17041 \pm 0.00175$ & $89.16 \pm 0.50$ & $0.07691 \pm 0.00245$ & $0.01311 \pm 0.00047$ \\
MPG/ESO 2.2-m & Sloan $i^{\prime}$ & $0.09239 \pm 0.00167$ & $0.17183 \pm 0.00161$ & $89.10 \pm 0.58$ & $0.07885 \pm 0.00134$ & $0.01355 \pm 0.00033$ \\
MPG/ESO 2.2-m & Sloan $z^{\prime}$ & $0.09391 \pm 0.00575$ & $0.17274 \pm 0.00226$ & $89.11 \pm 0.72$ & $0.08007 \pm 0.00091$ & $0.01383 \pm 0.00090$ \\
MPG/ESO 2.2-m & $J$ & $0.08937 \pm 0.00567$ & $0.16813 \pm 0.00424$ & $90.00 \pm 1.16$ & $0.07651 \pm 0.00476$ & $0.01286 \pm 0.00094$ \\
MPG/ESO 2.2-m & $H$ & $0.09393 \pm 0.00365$ & $0.17525 \pm 0.00472$ & $89.34 \pm 0.91$ & $0.07993 \pm 0.00296$ & $0.01401 \pm 0.00076$ \\
MPG/ESO 2.2-m & $K$ & $0.09067 \pm 0.00773$ & $0.16383 \pm 0.00809$ & $89.99 \pm 1.29$ & $0.07791 \pm 0.00673$ & $0.01276 \pm 0.00128$ \\
Euler 1.2-m & Gunn $r$ & $0.09694 \pm 0.00277$ & $0.16726 \pm 0.00270$ & $88.43 \pm 0.45$ & $0.08305 \pm 0.00223$ & $0.01389 \pm 0.00059$ \\
Trappist 0.60-m & Gunn $z$ & $0.09261 \pm 0.00251$ & $0.17079 \pm 0.00201$ & $88.64 \pm 0.49$ & $0.07910 \pm 0.00204$ & $0.01351 \pm 0.00047$ \\
Trappist 0.60-m & Gunn $z$ & $0.09464 \pm 0.00550$ & $0.16285 \pm 0.00230$ & $88.63 \pm 0.87$ & $0.08139 \pm 0.00467$ & $0.01325 \pm 0.00087$ \\
\hline Final results & & $\mathbf{0 . 0 9 2 8 3} \pm \mathbf{0 . 0 0 0 5 8}$ & $\mathbf{0 . 1 7 0 5 8} \pm \mathbf{0 . 0 0 0 5 7}$ & $\mathbf{8 8 . 9 1} \pm \mathbf{0 . 1 6}$ & $\mathbf{0 . 0 7 9 2 9} \pm \mathbf{0 . 0 0 0 4 6}$ & $\mathbf{0 . 0 1 3 5 4} \pm \mathbf{0 . 0 0 0 1 2}$ \\
\hline Triaud et al. (2013) & & - & $0.17126_{-0.00026}^{+0.00031}$ & $89.92_{-0.12}^{+0.07}$ & - & \\
\hline
\end{tabular}

Notes. The final parameters, given in bold, are the weighted means of the results for the datasets. Results from the discovery paper are included at the base of the table for comparison.

Table 5. Derived physical properties of the WASP-80 planetary system using empirical calibrations and each of five sets of theoretical models.

\begin{tabular}{lcccccc}
\hline \hline & $\begin{array}{c}\text { This work } \\
(\mathrm{dEB} \text { constraint })\end{array}$ & $\begin{array}{c}\text { This work } \\
(\text { Claret models })\end{array}$ & $\begin{array}{c}\text { This work } \\
\left(\mathrm{Y}^{2} \text { models }\right)\end{array}$ & $\begin{array}{c}\text { This work } \\
(\text { Teramo models })\end{array}$ & $\begin{array}{c}\text { This work } \\
(\text { VRSS models })\end{array}$ & $\begin{array}{c}\text { This work } \\
(\mathrm{DSEP} \text { models })\end{array}$ \\
\hline$K_{\mathrm{b}}\left(\mathrm{km} \mathrm{s}^{-1}\right)$ & $122.7 \pm 2.8$ & $126.9 \pm 1.3$ & $122.7 \pm 2.3$ & $122.5 \pm 2.2$ & $124.2 \pm 1.7$ & $123.6 \pm 1.8$ \\
$M_{\mathrm{A}}\left(M_{\odot}\right)$ & $0.589 \pm 0.040$ & $0.652 \pm 0.020$ & $0.588 \pm 0.034$ & $0.585 \pm 0.032$ & $0.610 \pm 0.024$ & $0.602 \pm 0.026$ \\
$R_{\mathrm{A}}\left(R_{\odot}\right)$ & $0.590 \pm 0.014$ & $0.611 \pm 0.007$ & $0.590 \pm 0.014$ & $0.589 \pm 0.011$ & $0.597 \pm 0.009$ & $0.595 \pm 0.010$ \\
$\log g_{\mathrm{A}}(\mathrm{cgs})$ & $4.666 \pm 0.011$ & $4.681 \pm 0.007$ & $4.666 \pm 0.006$ & $4.665 \pm 0.009$ & $4.671 \pm 0.008$ & $4.669 \pm 0.008$ \\
$M_{\mathrm{b}}\left(M_{\text {Jup }}\right)$ & $0.557 \pm 0.030$ & $0.596 \pm 0.020$ & $0.557 \pm 0.030$ & $0.555 \pm 0.025$ & $0.571 \pm 0.022$ & $0.566 \pm 0.023$ \\
$R_{\mathrm{b}}\left(R_{\text {Jup }}\right)$ & $0.981 \pm 0.024$ & $1.015 \pm 0.014$ & $0.981 \pm 0.023$ & $0.979 \pm 0.020$ & $0.993 \pm 0.016$ & $0.989 \pm 0.017$ \\
$\rho_{\mathrm{b}}\left(\rho_{\text {Jup }}\right)$ & $0.551 \pm 0.024$ & $0.533 \pm 0.021$ & $0.551 \pm 0.025$ & $0.552 \pm 0.023$ & $0.545 \pm 0.022$ & $0.547 \pm 0.022$ \\
$\Theta$ & $0.0668 \pm 0.0024$ & $0.0645 \pm 0.0020$ & $0.0668 \pm 0.0025$ & $0.0669 \pm 0.0023$ & $0.0660 \pm 0.0021$ & $0.0663 \pm 0.0021$ \\
$a(\mathrm{AU})$ & $0.03464 \pm 0.00079$ & $0.03583 \pm 0.00037$ & $0.03463 \pm 0.00064$ & $0.03457 \pm 0.00062$ & $0.03506 \pm 0.00047$ & $0.03490 \pm 0.00050$ \\
\hline
\end{tabular}


Table 6. Final physical properties of the WASP-80 planetary system, compared with results from Triaud et al. (2013).

\begin{tabular}{llcc}
\hline \hline & & This work (final) & Triaud et al. (2013) \\
\hline Stellar mass & $M_{\mathrm{A}}\left(M_{\odot}\right)$ & $0.596 \pm 0.032 \pm 0.014$ & $0.57_{-0.05}^{+0.05}$ \\
Stellar radius & $R_{\mathrm{A}}\left(R_{\odot}\right)$ & $0.593 \pm 0.011 \pm 0.005$ & $0.571_{-0.016}^{+0.016}$ \\
Stellar surface gravity & $\log g_{\mathrm{A}}(\mathrm{cgs})$ & $4.6678 \pm 0.0077 \pm 0.0034$ & $4.689_{-0.013}^{+0.012}$ \\
Stellar density & $\rho_{\mathrm{A}}\left(\rho_{\odot}\right)$ & $2.862 \pm 0.050$ & $3.117_{-0.020}^{+0.021}$ \\
Planetary mass & $M_{\mathrm{b}}\left(M_{\mathrm{Jup}}\right)$ & $0.562 \pm 0.025 \pm 0.009$ & $0.554_{-0.039}^{+0.030}$ \\
Planetary radius & $R_{\mathrm{b}}\left(R_{\mathrm{Jup}}\right)$ & $0.986 \pm 0.020 \pm 0.008$ & $0.952_{-0.027}^{+0.026}$ \\
Planetary surface gravity & $g_{\mathrm{b}}\left(\mathrm{m} \mathrm{s}^{-2}\right)$ & $14.34 \pm 0.46$ & $15.07_{-0.42}^{+0.45}$ \\
Planetary density & $\rho_{\mathrm{b}}\left(\rho_{\mathrm{Jup}}\right)$ & $0.549 \pm 0.023 \pm 0.004$ & $0.554_{-0.039}^{+0.030}$ \\
Planetary equilibrium temperature & $T_{\text {eq }}^{\prime}(\mathrm{K})$ & $825 \pm 20$ & $\sim 800$ \\
Safronov number & $\Theta$ & $0.0665 \pm 0.0023 \pm 0.0005$ & - \\
Orbital semimajor axis & $a(\mathrm{au})$ & $0.03479 \pm 0.00062 \pm 0.00027$ & $0.0346_{-0.011}^{+0.0008}$ \\
\hline
\end{tabular}

Notes. Separate statistical and systematic error bars are given for the results from the current work.

predicted by the various model sets (see Fig. 4 in Southworth 2010).

Theoretical models prefer a larger age for the system than is reasonable based on the activity level of the host star. There are several cases (e.g. CoRoT-2 and HD 189733), where a planethosting star displays a much higher magnetic activity level than expected for their age (Poppenhaeger \& Wolk 2013). A similar behaviour has also been suggested in the case of Qatar-1 (Covino et al. 2013). Several studies pointed out that a close-in hot-Jupiter can produce different effects on its parent star. Tidal forces can increase the rotational velocity of the star (Pont 2009). The effect of the magnetized stellar wind, which causes loss of angular momentum, is inhibited by the planet (Lanza et al. 2010; Cohen et al. 2010). Both processes make the star appear younger than it is. This situation can be investigated through a detailed spectroscopic analysis of the host star to refine its measured atmospheric parameters, and a study of its X-ray luminosity, which is known to decline with stellar age (e.g. Wright et al. 2011). In the meantime we do not report an age measurement for the system.

For the final system properties we took the unweighted means of the four concordant model sets and calculated systematic error bars based on the interagreement between them. A comparison between our final values and those found by Triaud et al. (2013) is given in Table 6.

\section{Variation of the planetary radius with wavelength}

WASP-80 b is a good target for studies of the planetary atmosphere due to the low surface gravity, deep transit, and bright host star. However, its moderate equilibrium temperature $\left(T_{\mathrm{eq}}^{\prime}=\right.$ $825 \pm 20 \mathrm{~K}$ ) indicates that the planet should belong to the $\mathrm{pL}$ class, as suggested by Fortney et al. (2008). We therefore do not expect a big variation of the measured planet radius with wavelength. Our GROND data, however, are very well suited to investigating this possibility as they cover many passbands.

We have measured the ratio of the planetary and stellar radius, $k$, in the GROND light curves. Figure 6 shows the result as a function of wavelength. The vertical error bars represent the relative errors in the measurements (i.e. neglecting sources of error which affect all light curves equally), and the horizontal error bars show the FWHM transmission of the passbands used. Due to the very large uncertainty, the values of $k$ measured in the $H$ and $K$ bands were ignored. For the $J$ band, following Southworth et al. (2012) and Mancini et al. (2013b), we refitted the data with all parameters fixed to the final values given in Table 4, with the exception of $k$. This approach maximizes the precision of estimations of the planet/star radius ratio. As our final value for $k$ in the $J$ band, we got $k=0.1695 \pm 0.0028$.

The $k$ found for the data from the Danish Telescope is also shown in green, and is a good match with the results for the GROND $i^{\prime}$ data. For illustration, Fig. 6 also shows the predictions from a model atmosphere calculated by Fortney et al. (2010) for a Jupiter-mass planet with gravity $g_{\mathrm{b}}=10 \mathrm{~m} \mathrm{~s}^{-2}$, a base radius of $1.25 R_{\mathrm{Jup}}$ at $10 \mathrm{bar}$, and $T_{\mathrm{eq}}^{\prime}=750 \mathrm{~K}$. The opacity of strong-absorber molecules, such as gaseous titanium oxide ( TiO) and vanadium oxide (VO), was removed from the model. Our experimental points are in agreement with the prominent absorption features of the model and, being compatible with a flat transmission spectrum, do not indicate any large variation of the WASP-80 b's radius.

\section{Summary and conclusions}

The WASP-80 system contains a low-mass star which is at the border of the $M$ spectral class, and a transiting hot Jupiter. It is one of only two systems containing an M-dwarf and a Jupiter-size object, the other being the much fainter Kepler45. The brightness and small radius of the host star, which means the planetary transits are deep, and the low surface gravity of the planet make it a very important object for studying the atmospheric characteristics of irradiated gas giant planets.

We present eight light curves of one transit event, taken simultaneously using two telescopes, in the Bessell $I$, Sloan $g^{\prime}$, $r^{\prime}, i^{\prime}, z^{\prime}$, and the NIR $J H K$ passbands. We find a good agreement for the ratio of the planetary to stellar radius determined from our optical light curves, which means that we do not detect any opacity-induced changes in planetary radius. We model all available optical transit light curves and use these results to determine the physical properties of the system. Our values are consistent with previous measurements, although our value for the planetary radius is $1 \sigma$ larger than that in the discovery paper.

We also present a spectrum of WASP-80 covering the $\mathrm{Ca} \mathrm{H}$ and $\mathrm{K}$ lines, which shows strong emission in the line cores. We measure a chromospheric activity indicator of $\log R_{\mathrm{HK}}^{\prime}=-4.495$, which makes WASP-80 one of the most 


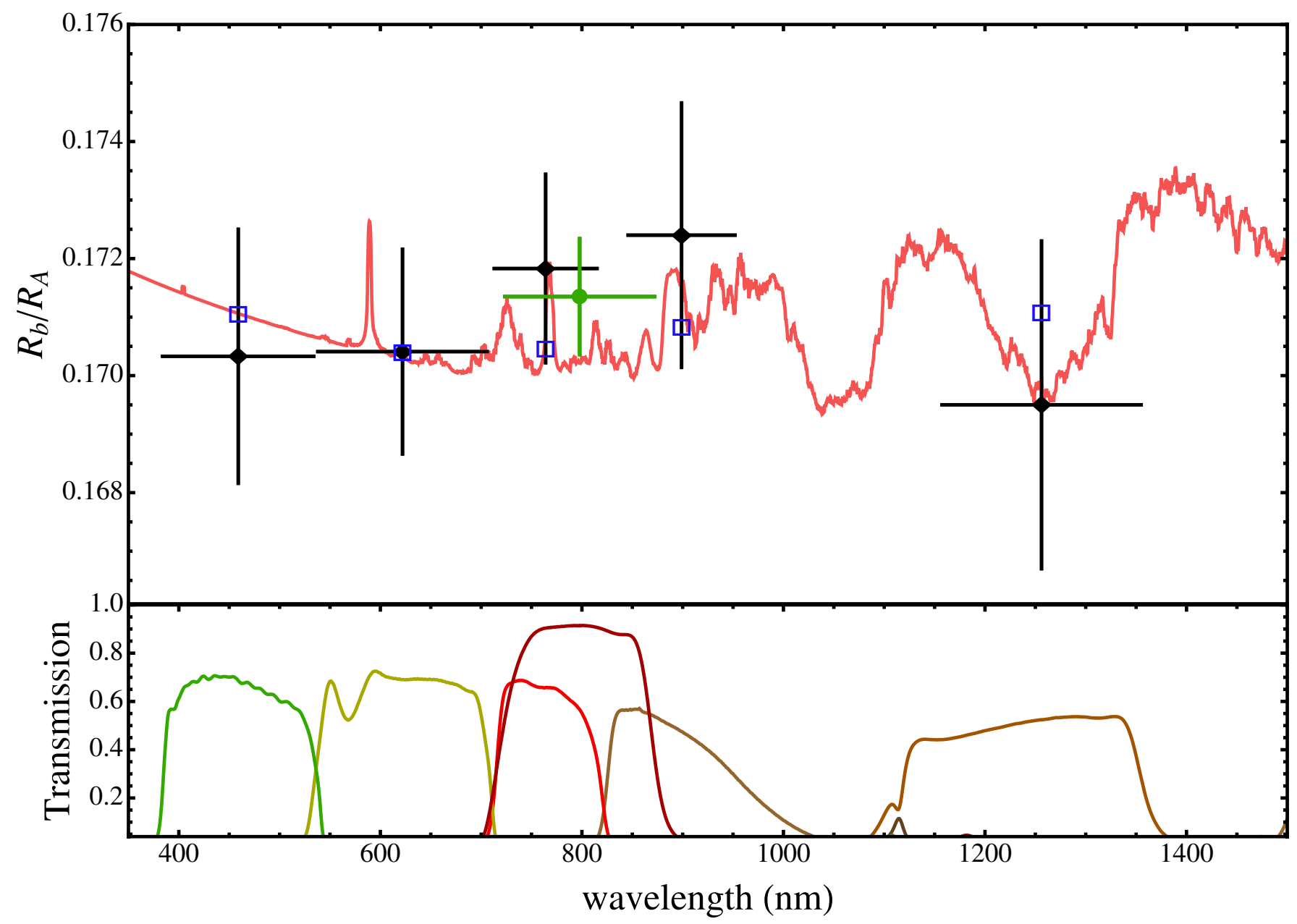

Fig. 6. Variation of the planetary radius, in terms of planet/star radius ratio, with wavelength. The black diamonds are from the transit observations performed with GROND while the green point is from the same transit observed using the Danish Telescope. The vertical bars represent the errors in the measurements and the horizontal bars show the FWHM transmission of the passbands used. The observational points are compared with a synthetic spectrum (see text for details). Transmission curves for the Bessel $I$ filter and the total efficiencies of the GROND filters are shown in the bottom panel. The blue boxes indicate the predicted values for the model integrated over the passbands of the observations.

active planet hosts known. This implies strong magnetic activity and the presence of starspots, although we see no evidence in our high-precision photometry for starspot crossing events. If starspots exist on the surface of WASP-80 A, they are likely small, numerous and evenly distributed on the stellar photosphere.

Triaud et al. (2013) do not report a conclusive measurement of $v \sin i_{\star}$. This is because the one obtained by the broadening of the spectral lines is incompatible with the one derived from fitting the Rossiter-McLaughlin effect, suggesting that the planet's orbital spin could be very inclined. Since the stellar rotation period is highly uncertain, an estimate of the stellar age based on gyrochronology is not possible. The age constraints implied by the stellar models in Sect. 4 are older than the strong activity of WASP-80 A suggests (see Pace 2013, Fig. 1). A possible explanation is that the stellar activity is enhanced by its planet. However, an estimation of the stellar age based on theoretical models is very uncertain because $\mathrm{M}$ stars evolve very slowly during their main-sequence lifetime. X-ray observations may help explain this discrepancy.

A detailed characterisation of the atmosphere of WASP-80 b could be performed using transmission spectroscopy. We caution that such investigations should be based on simultaneous observations in order to avoid complications due to starspot activity.
Acknowledgements. This paper is based on observations collected with the MPG/ESO 2.2-m and the Danish 1.54-m telescopes, both located at ESO La Silla, Chile. Operation of the MPG/ESO 2.2-m telescope is jointly performed by the Max Planck Gesellschaft and the European Southern Observatory. Operation of the Danish telescope is based on a grant to U.G.J. by the Danish Natural Science Research Council (FNU). GROND was built by the highenergy group of MPE in collaboration with the LSW Tautenburg and ESO, and is operated as a PI-instrument at the MPG/ESO 2.2-m telescope. J.S. (Keele) acknowledges financial support from STFC in the form of an Advanced Fellowship. C.S. received funding from the European Union Seventh Framework Programme (FP7/2007-2013) under grant agreement No. 268421. M.R. acknowledges support from FONDECYT postdoctoral fellowship $\mathrm{N}^{\circ} 3120097$. S.-H.G. and X.-B.W. would like to thank the financial support from National Natural Science Foundation of China (No.10873031) and Chinese Academy of Sciences (project KJCX2-YW-T24). O.W. thanks the Belgian National Fund for Scientific Research (FNRS). J.S. and O.W. acknowledge support from the Communauté francaise de Belgique - Actions de recherche concertées Académie universitaire Wallonie-Europe. K.A., M.D. and M.H. acknowledge grant NPRP-09-476-1-78 from the Qatar National Research Fund (a member of Qatar Foundation). The following internet-based resources were used in research for this paper: the ESO Digitized Sky Survey; the NASA Astrophysics Data System; the SIMBAD data base operated at the CDS, Strasbourg, France; and the arXiv scientific paper preprint service operated by Cornell University.

\section{References}

Bean, J. L., Désert, J.-M., Kabath, P., et al. 2011, ApJ, 743, 92 Berdyugina, S. V. 2005, LRSP, 2, 8 
Berta, Z. K., Charbonneau, D., Désert, J.-M., et al. 2012, ApJ, 747, 35 Bonfils, X., Delfosse, X., Udry, S., et al. 2013, 549, A109

Carter, J. A., \& Winn, J. N. 2009, ApJ, 704, 51

Claret, A. 2004a, A\&A, 424, 919

Claret, A. 2004b, A\&A, 428, 1001

Cohen, O., Drake, J. J., Kashyap, V. L., et al. 2010, ApJ, 723, L64

Colòn, K. D., \& Gaidos, E. 2013, ApJ, 776, 49

Covino, E., Esposito, M., Barbieri, M., et al. 2013, A\&A, 554, A28

Croll, B., Albert, L., Jayawardhana, R., et al. 2011, ApJ, 736, 78

Crossfield, I. J. M., Barman, T., \& Hansen, B. M. S. 2011, ApJ, 736, 132

Crossfield, I. J. M., Barman, T., Hansen, B. M. S., \& Howard, A. 2013, A\&A, 559, A33

de Mooij, E. J. W., Brogi, M., de Kok, R. J., et al. 2012, A\&A, 538, A46

de Mooij, E. J. W., Brogi, M., de Kok, R. J., et al. 2013, ApJ, 771, 109

Demarque, P., Woo, J.-H., Kim, Y.-C., \& Yi, S. K. 2004, ApJS, 155, 667

Dominik, M., Jørgensen, U. G. , Rattenbury, N. J., et al. 2010, AN, 331, 671

Dotter, A., Chaboyer, B., Jevremović, D., et al. 2008, ApJS, 178, 89

Dressing, C. D., \& Charbonneau, D. 2013, ApJ, 767, 95

Enoch, B., Collier Cameron, A., Parley, N. R., \& Hebb, L. 2010, A\&A, 516, A33

Fortney, J. J., Lodders, K., Marley, M. S., \& Freedman R. S. 2008, ApJ, 678, 1419

Fortney, J. J., Shabram, M., \& Showman, A. P. 2010, ApJ, 709, 1396

Fraine, J. D., Deming, D., Gillon, M., et al. 2013, ApJ, 765, 127

Gibson, N. P., Pollacco, D., Simpson, E. K., et al. 2008, A\&A, 492, 603

Gibson, N. P., Pont, F., \& Aigrain, S. 2011, MNRAS, 411, 2199

Gillon, M., Pont, F., Moutou, C., et al. 2006, A\&A, 459, 249

Greiner, J., Bornemann, W., Clemens, C., et al. 2008, PASP, 120, 405

Irwin, J., Buchhave, L., Berta, Z. K., et al. 2010, ApJ, 718, 1353

Jackson, R. J., \& Jeffries, R. D. 2012, MNRAS, 423, 2966

Jackson, R. J., \& Jeffries, R. D. 2013, MNRAS, 431, 1883

Johnson, J. A., Aller, K. M., Howard, A. W., \& Crepp, J. R. 2010, PASP, 122, 905
Johnson, J. A., Apps, K., Gazak, J. Z., et al. 2011, ApJ, 730, 79 Johnson, J. A., Gazak, J. Z., Apps, K., et al. 2012, AJ, 143, 111 Knutson, H. A., Madhusudhan, N., Cowan, N. B., et al. 2011, ApJ, 735, 27 Lanza, A. F. 2010, A\&A, 512, A77

Mancini, L., Southworth, J., Ciceri, S., et al. 2013a, A\&A, 551, A11

Mancini, L., Nikolov, N., Southworth, J., et al. 2013b, MNRAS, 430, 2932

Mancini, L., Ciceri, S., Chen, G., et al. 2013c, MNRAS, 436, 2

Marsh, T. R. 1989, PASP, 101, 1032

Murgas, F., Pallé, E., Cabrera-Lavers, A., et al. 2012, A\&A, 544, A41

Narita, N., Fukui, A, Ikoma, M., et al. 2013, ApJ, 773, 144

Nascimbeni, V., Cunial, A., Murabito, S., et al. 2013, A\&A, 549, A30

Noyes, R. W., Hartmann, L. W., Baliunas, S. L., et al. 1984, ApJ, 279, 763

Pace, G. 2013, A\&A, 551, L8

Pietrinferni, A., Cassisi, S., Salaris, M., \& Castelli, F. 2004, ApJ, 612, 168

Pont, F. 2009, MNRAS, 396, 1789

Pont, F., Gilliland, R. L., Knutson, H., et al. 2009, MNRAS, 393, L6

Poppenhaeger, K., \& Wolk, S. J. 2013, Proc. IAUS 302: Magnetic Fields Throughout Stellar Evolution, in press [arXiv: 1309. 6356]

Southworth, J. 2008, MNRAS, 386, 1644

Southworth, J. 2009, MNRAS, 394, 272

Southworth, J. 2010, MNRAS, 408, 1689

Southworth, J. 2012, MNRAS, 426, 1291

Southworth, J., Hinse, T. C., Jørgensen, U. G., et al. 2009, MNRAS, 396, 1023

Southworth, J., Mancini, L., Maxted, P. F. L., et al. 2012, MNRAS, 422, 3099

Stevenson, K. B., Harrington, J., Nymeyer, S., et al. 2010, Nature, 464, 161

Tregloan-Reed, J., Southworth, J., \& Tappert, C. 2013, MNRAS, 428, 3671

Triaud, A. H. M. J., Anderson, D. R., Collier Cameron, A., et al. 2013, A\&A, 551, A80

VandenBerg, D. A., Bergbusch, P. A., \& Dowler, P. D. 2006, ApJS, 162, 375

Vaughan, A. H., Preston, G. W., \& Wilson, O. C. 1978, PASP, 90, 267

West, A. A., Hawley, S. L., \& Bochanski, J. J. 2008, ApJ, 135, 785

Winn, J. N., Holman, M. J., Torres, G., et al. 2008, ApJ, 683, 1076

Wright, N. J., Drake, J. J., Mamajek, E. E., \& Henry, G. W. 2011, ApJ, 743, 48 